\title{
DETECTING CHANGES IN USER-CENTERED MUSIC QUERY STREAMS
}

\author{
Hua-Fu Li ${ }^{* a}$, Man-Kwan Shan ${ }^{b}$, and Suh-Yin Lee ${ }^{a}$ \\ ${ }^{a}$ Department of Computer Science, National Chiao-Tung University, Hsinchu 300, Taiwan \\ ${ }^{b}$ Department of Computer Science, National Chengchi University, Taipei 116, Taiwan \\ \{hfli, sylee\}@csie.nctu.edu.tw; mkshan@cs.nccu.edu.tw
}

\begin{abstract}
In this paper, we propose an efficient algorithm, called MQSchange (changes of Music Query Streams), to detect the changes of maximal melody structures in user-centered music query streams. Two music melody structures (set of chord-sets and string of chord-sets) are maintained and four melody structure changes (positive burst, negative burst, increasing change and decreasing change) are monitored in a new data structure MSC-list (a list of Music Structure Changes). Experiments show that MQS-change algorithm is an online, single-pass approach to detect the changes of music melody structures over continuous music query streams.
\end{abstract}

\section{INTRODUCTION}

Recently, database and data mining communities have been focused on a new data model, where data arrives in the form of continuous streams. It is often refer to data streams or streaming data. Many applications generate large amount of data streams in real time, such as sensor data generated from sensor networks, transaction flows in retail chains, Web click and record streams in Web applications, performance measurement in network monitoring and traffic management, call records in telecommunications, etc. Mining streaming data differs from mining traditional static data sets in two main aspects [2] [6]:

- The volume of a continuous stream over its lifetime could be huge and fast changing.

- The queries require timely answers, and the response time is short.

Hence, it is not possible to store all the data in main memory or even in secondary storage. This motivates the design for inmemory summary data structure with small memory footprints that can support both one-time and continuous queries. In other words, online algorithms of mining data streams have to sacrifice the correctness of their analysis results by allowing some counting errors, i.e., approximate results, and only have one pass over the data.

Mining music data is one of the most important research issues in data mining. Although several techniques have been developed recently for discovering and analyzing the content of static music data [3][7][12][13], new techniques are needed to analyze and discover the content of streaming music data. Li et al. [9][10] proposed efficient single-pass algorithms to find maximal frequent melody structures and closed frequent melody structures over continuous music query streams. The problem comes from the context of online music-downloading services (such as iTunes, Kuro [14] and KKBOX [15]), where the streams in question are streams of queries, i.e., musicdownloading requests, sent to the server, and we are interested in finding the useful music melody structures requested by most customers during some period of time. With the computation model of music melody streams presented in Fig. 1 , the melody stream processor and the summary data structure are two major components in such a streaming environment. The user query processor receives user queries in the form of <Timestamp, Customer-ID, Music-ID>, and then transforms the queries into music data (i.e., melody sequences) in the form of <Timestamp, Customer ID, Music-ID, Melody-Sequence> by querying the music database. Note that the buffer can be optionally set for temporary storage of recent music melodies from the music melody streams.

With data streams, people are more often interested in mining queries such like "Compared to the history, what are the distinct features of the current status?", " What are the most popular melody structures in the last four hours?" and "What are the relatively stable factors over time?" To answer the above queries, we have to examine the changes of streaming data [5][11]. Hence, this paper studies a new problem of how to detect the changes of maximal melody structures over user-centered music query streams. An efficient single-pass algorithm MQS-change is proposed to detect the changes of music query streams. Experiments show that the proposed algorithm is an effective algorithm to detect the changes in data streams efficiently.

The remainder of the paper is organized as follows. The problem is defined in Section 2. In Section 3, we describe the design of the MQS-change algorithm for detecting changes of music query streams. Experiments are discussed in Section 4. Finally, we conclude our work in Section 5.

*Corresponding author. Fax: 886-3-5721490. E-mail: hfli@csie.nctu.edu.tw 


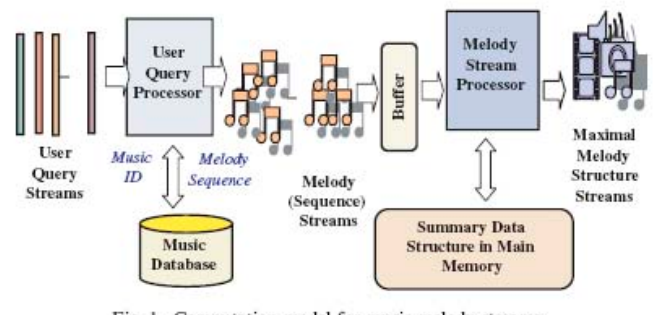

Fig. 1. Computation model for music melody streams.

\section{PROBLEM DEFINITION}

In this section, we describe several features of music data used in this paper and define the problem of detecting changes of user-centered music query streams. For the basic terminologies on music, we refer to [8][12].

Definition 1 A chord is the sounding combination of three or more notes at the same time. A note is a single symbol on a musical score, indicating the pitch and duration of what is to be sung and played. A chord-set is a set of chords.

Definition 2 The type I melody structure is represented as a set of chord-sets.

Definition 3 The type II melody structure is represented as a string of chord-sets.

Definition 4 Let $\Psi=\left\{i_{1}, i_{2}, \ldots, i_{n}\right\}$ be a set of chord-sets, called items for simplicity. A melody sequence $S$ with $m$ chord-sets is denoted by $S_{m}=\left\langle x_{1} x_{2} \ldots x_{m}\right\rangle$, where $x_{i} \in \Psi, \forall i=1,2, \ldots, m$. A basic window $w$ is consecutive subsequence of $t$ melody sequences. The size of a basic window $|w|$ is $t$.

Definition 5 A music melody stream (MMS) is an infinite sequence of basic windows, where each window $w_{i}$ is associated with a window identifier $i$, and $z$ is the identifier of the "latest'" window $w_{z}$, i.e., MMS $=\left[w_{1}, w_{2}, \ldots, w_{z}\right)$. The current length of MMS, written as $|\mathrm{MMS}|$, is $z t$, i.e., $|\mathrm{MMS}|=$ $\left|w_{1}\right|+\left|w_{2}\right|+\cdots+\left|w_{z}\right|$. These windows arrive in some order (implicitly by arrival time or explicitly by timestamp), and may be seen only once.

Definition 6 A set $Y \subseteq \Psi$ is called an itemset, i.e., a set of chord-sets. A k-itemset is represented by $\left(y_{1} y_{2} \cdots y_{k}\right)$. The support of an itemset $Y$, denoted as $\sup (Y)$, is the number of melody sequences containing $Y$ as a subset in the MMS so far. An itemset $Y$ is frequent if $\sup (Y) \geq s \cdot \mid \mathrm{MMS|}$, where $s$ is a userdefined minimum support threshold in the range of $[0,1]$.

Definition 7 A string $Z$ is called an item-string, i.e., a string of chord-sets. A $k$-item-string is represented by $\left\langle z_{1} z_{2} \quad \ldots \quad z_{k}\right\rangle$, where $z_{i} \in \Psi, \forall i=1,2, \ldots, k$. The support of an item-string $Z$, denoted as $\sup (Z)$, is the number of melody sequences containing $Z$ as a substring in the MMS so far. An item-string is frequent if $\sup (Z) \geq s \cdot|\mathrm{MMS}|$.

Definition 8 A frequent itemset is called a maximal frequent itemset (MFI) if it is not a subset of any other frequent itemsets. Definition 9 A frequent item-string is called a maximal frequent item-string (MFS) if it is not a substring of any other item-strings.

Definition 10 A maximal frequent itemset $P$ is called positive itemset burst $(\mathbf{P I B})$ if its $\sup (P)^{\mathrm{z}}-\sup (P)^{\mathrm{z}-1} \geq \partial_{\mathrm{MFI}}$, where $\partial_{\mathrm{MFI}}$ is a user-specified itemset burst threshold in the range of $[0,1]$, $\sup (P)^{\mathrm{Z}}$ is the estimated support of $P$ from window $w_{1}$ to window $w_{z}$.

Definition 11 A maximal frequent itemset $P$ is called negative itemset burst $(\mathrm{NIB})$ if its $\sup (P)^{\mathrm{z}-1}-\sup (P)^{\mathrm{z}} \geq \partial_{\mathrm{MFI}}$

Definition 12 A maximal frequent item-string $Q$ is called positive item-string burst (PSB) if its $\sup (Q)^{\mathrm{z}}-\sup (Q)^{\mathrm{z}-1} \geq$ $\partial_{\mathrm{MFS}}$, where $\partial_{\mathrm{MFS}}$ is a user-specified item-string burst threshold in the range of $[0,1], \sup (Q)^{\mathrm{z}}$ is the estimated support of $Q$ from window $w_{1}$ to window $w_{z}$.

Definition 13 A maximal frequent item-string $Q$ is called negative item-string burst (NSB) if its $\sup (Q)^{\mathrm{z}-1}-\sup (Q)^{\mathrm{z}} \geq$ $\partial_{\mathrm{MFs}}$.

Definition 14 A maximal frequent itemset $P$ is called increasing changed itemset $(\mathrm{ICI})$ if $\partial_{\mathrm{MFI}}>\left(\sup (P)_{i+1}-\sup (\mathrm{P})_{i}\right)$ $\geq \varepsilon_{\mathrm{MFI}}, \forall i, i=z-h_{1}+1, z-h_{1}+2, \ldots, z$, where $\varepsilon_{\mathrm{MFI}}$ is a userspecified increasing changed itemset threshold in the range of $[0,1]$, and $h_{1}$ is a number of basic windows defined by user.

Definition 15 A maximal frequent item-string $Q$ is called increasing changed item-string (ICS) if $\partial_{\mathrm{MFS}}>\left(\sup (Q)_{j+1}-\right.$ $\left.\sup (Q)_{j}\right) \geq \varepsilon_{\mathrm{MFS}}, \forall j, j=z-h_{2}+1, z-h_{2}+2, \ldots, z$, where $\varepsilon_{\mathrm{MFS}}$ is a user-specified increasing changed item-string threshold in the range of $[0,1]$, and $h_{2}$ is a number of basic windows defined by user.

Definition 16 A maximal frequent itemset $P$ is called decreasing changed itemset (DCI) if $\partial_{\mathrm{MFI}}>\left(\sup (Q)_{j}-\right.$ $\left.\sup (Q)_{j+1}\right) \geq \gamma_{\mathrm{MFI}}, \forall j, j=z-h_{1}+1, z-h_{1}+2, \ldots, z$, where $\gamma_{\mathrm{MFI}}$ is a user-specified decreasing changed item-string threshold in the range of $[0,1]$, and $h_{1}$ is a number of basic windows defined by user.

Definition 17 A maximal frequent item-string $Q$ is called decreasing changed item-string (DCS) if $\partial_{\mathrm{MFS}}>\left(\sup (Q)_{j}-\right.$ $\left.\sup (Q)_{j+1}\right) \geq \gamma_{\mathrm{MFS}}, \forall j, j=z-h_{2}+1, z-h_{2}+2, \ldots, z$, where $\gamma_{\mathrm{MFS}}$ is a user-specified decreasing changed item-string threshold in the range of $[0,1]$, and $h_{2}$ is a number of basic windows defined by user.

Problem Definition Given a MMS, $s, \partial_{\mathrm{MFI}}, \partial_{\mathrm{MFS}}, \varepsilon_{\mathrm{MFI}}, \varepsilon_{\mathrm{MFS}}$, $\gamma_{\mathrm{MFI}}$, and $\gamma_{\mathrm{MFS}}$, the problem of detecting changes in usercentered music query streams is to maintain the set of MFI and MFS, and to detect the set of PIB, NIB, PSB, NSB, ICI, ICS, DCI, and DCS, by one scan of a continuous user-centered music query stream.

\section{DETECTING CHANGES IN USER-CENTERED MUSIC QUERY STREAMS}

In this section, a new summary data structure MSC-list (a list of Music Structure Changes) is developed to maintain the essential information about MFI, MFS, PIB, NIB, PSB, NSB, ICI, ICS, DCI, and DCS with their supports embedded in the individual window of the current MMS. An online, single-pass algorithm MQS-change (changes of Music Query Streams) is proposed to mine the changes from user-centered music query streams. 


\subsection{A New Summary Data Structure MSC-list}

MSC-list consists of two temporal lists, MFI-list and MFS-list, where MFI-list is a list of entries which contains current maximal frequent itemsets, and MFS-list is a list of entries which maintains maximal frequent item-strings so far. Each entry of MFI-list consists of two fields: pattern-id $Y$ and support-list Y.support-list, where pattern-id is a unique identifier of this maximal frequent itemset, and support-list is composed of a list of $(\sup (Y), i)$, where $i$ is the window identifier of window $w_{i}$ containing the itemset $e$. For example, an entry <abcd, $(30 \%, 1),(37 \%, 2),(46 \%, 3),(70 \%, 4)>$ of MFI-list indicates that the itemset $a b c d$ is a maximal frequent itemset and its estimated support is $30 \%$ in window $w_{1}, 37 \%$ in $w_{2}, 46 \%$ in $w_{3}$, and $70 \%$ in $w_{4}$. Assume that the $\partial_{\mathrm{MFI}}$ is 0.2 (i.e., $20 \%$ ), $\varepsilon_{\mathrm{MFI}}=0.05$ (i.e., $5 \%$ ), and $h_{1}$ be 3 (i.e., 3 consecutive windows). Hence, the pattern abcd is an increasing changed itemset from windows $w_{1}$ to $w_{3}$, and has a positive itemset burst in window $w_{4}$. Eeach entry of MFS-list also consists of two fields: pattern-id $Z$ and support-list Z.support-list, where pattern-id is a unique identifier of this maximal frequent itemstring, and support-list is composed of a list of $(\sup (Z), i)$, where $i$ is the identifier of window $w_{i}$ containing the item-string $Z$. In the following, we use the term maximal frequent pattern (MFP) to substitute the maximal frequent itemset and maximal frequent item-string.

Two operations are used to maintain the MSC-list:

(1) Update MSC-list: For each entry <pattern-id, supportlist> of MSC-list, MQC-change algorithm updates the support-list of this entry, i.e., append a new support record to the support-list. If there are changes happen (according to the definition 11 through definition 17), the pattern is inserted into a temporal change output quеие (TCOQ). If an entry $e$ is not a MFP, i.e., $\sup (e)<s \cdot \mid \mathrm{MMSI}$, the entry is deleted from the current MSC-list.

(2) New MSC-list: if MQC-change find a maximal frequent pattern $P$ from the current window $w_{z}$ and $P \notin$ MSC-list, and $\sup (P) \geq$ s.t, where $s$ is the minimum support threshold, and $t$ is the window size, a new entry of the form $<P,(\sup (P), z)>$, where $z$ is the current window identifier, is created in the current MSC-list.

\subsection{The Proposed Algorithm}

The proposed MQS-change algorithm is composed of four steps. First, MQS-change repeatedly reads a window of melody sequences into available main memory. Second, the maximal frequent itemsets and maximal frequent item-strings in the current window are mined using $\mathrm{MMS}_{\mathrm{LMS}}$ algorithm [10], and added into MSC-list with their potential supports computed. Third, the set of MFIs and MFSs are maintained in the current MSC-list, and the changes are verified by MQS-change. Finally, MQS-change will return the changed patterns immediately if the user-centered music query stream has a change.

\subsubsection{Description of $M M S_{L M S}$ Algorithm}

We use an illustrative example to describe the main idea of $\mathrm{MMS}_{\mathrm{LMS}}$ algorithm. Let a window $w_{j}$ of MMS be <acdef>, $<a b e>,<c e f>,<a c d f>,<c e f>$, and $<d f>$, and minimum support threshold $s$ be 0.5 , where $a, b, c, d, e$, and $f$ are chord-sets.

First, $\mathrm{MMS}_{\mathrm{LMS}}$ algorithm projects each melody sequence into a set of item-suffix melody sequences. For example, the first melody sequence $<$ acdef $>$ is projected into five item-suffix melody sequences, i.e., $\langle f\rangle,<e f\rangle,<\operatorname{def}\rangle,<\operatorname{cdef}\rangle$, and $<$ acdef $>$.

Second, these item-suffix melody sequences are inserted into a prefix tree-based summary data structure. The result of processing first melody sequence $<$ acdef $>$ is shown in Fig. 2.

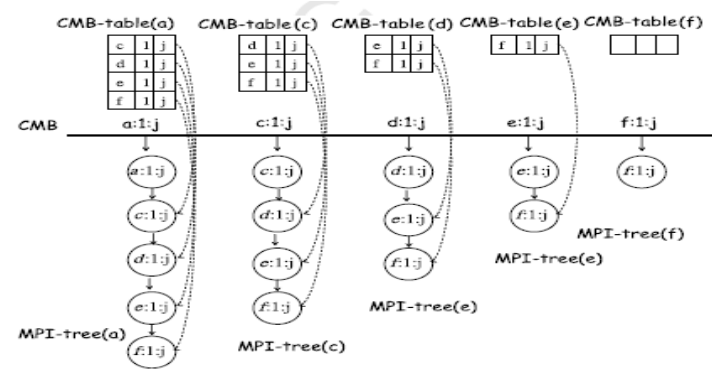

Fig. 2. Processing first melody sequence $<$ acdef $>$ by $\mathrm{MMS}_{\mathrm{LMS}}$

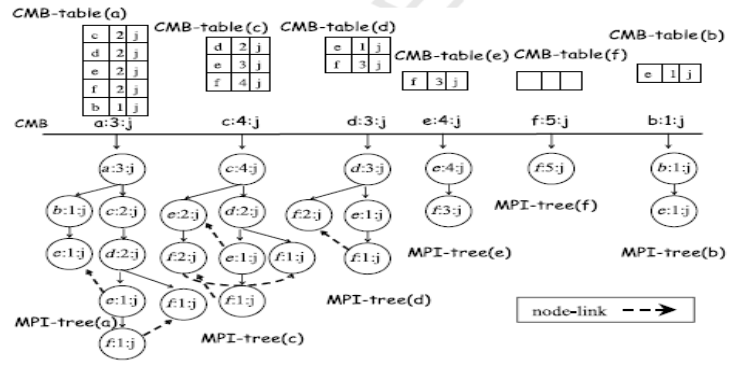

Fig. 3. Processing the window $w_{i}$ by $\mathrm{MMS}_{\mathrm{LMS}}$

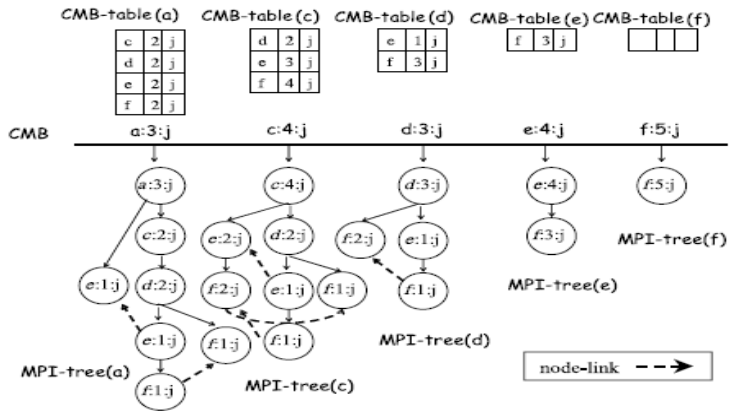

Fig. 4. Pruning infrequent information by $\mathrm{MMS}_{\mathrm{LMS}}$

After processing the window $w_{j}$ (the result is shown in Fig. 3), $\mathrm{MMS}_{\mathrm{LMS}}$ prunes infrequent patterns, i.e., item $b$ and its super-patterns, by traversing the summary data structure proposed by $\mathrm{MMS}_{\mathrm{LMS}}$ algorithm. The result after pruning infrequent patterns is shown in Fig. 4.

Finally, $\mathrm{MMS}_{\mathrm{LMS}}$ algorithm uses top-down maximal pattern discovery technique to find the set of MFIs, $(a),(c e f)$ and $(d f)$, and MFSs, $(a),(c),(d)$, and $(e f)$. More detail about the $\mathrm{MMS}_{\mathrm{LMS}}$ algorithm can be found in [10].

\subsubsection{Algorithm MQS-change}

The MQS-change algorithm is shown in Fig. 5. 


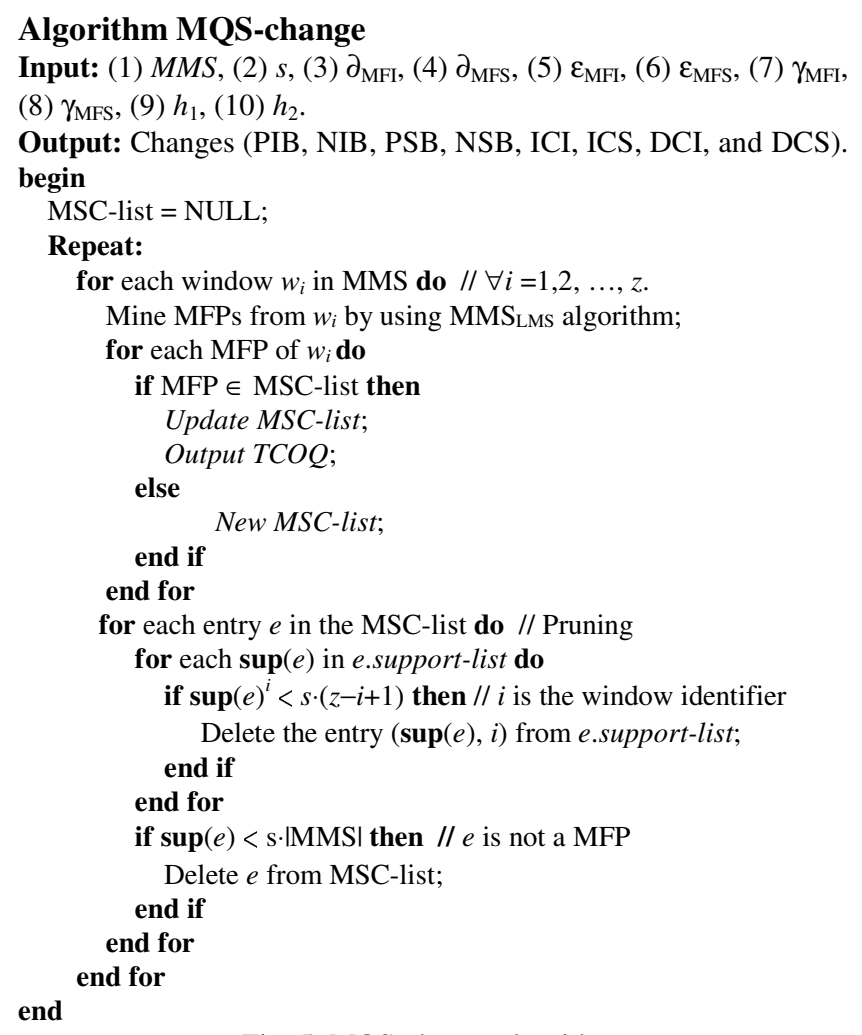

Fig. 5. MQS-change algorithm

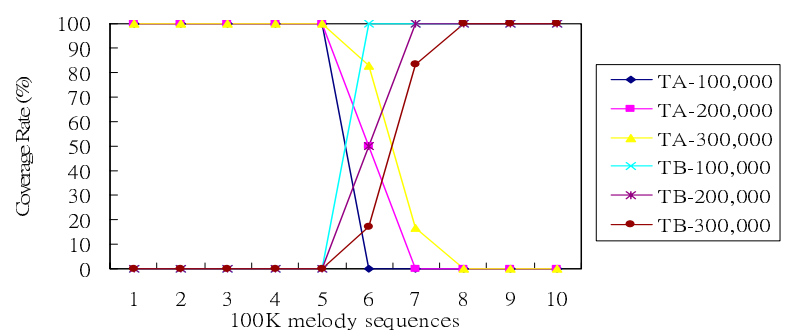

Fig 6. Coverage rate for T5.I4.D1000K-AB

\section{PERFORMANCE EVALUATION}

In this section, the performance of MQS-change algorithm is analyzed by a synthetic music query stream T5.I4.D1000K-AB, where three parameters denote the average melody sequence size $(T)$, the average maximal frequent pattern size $(I)$, and the total music melody sequences $(D)$, respectively. The data is generated by the IBM synthetic data generator proposed by Agrawal and Srikant [1]. T5.I4.D1000K-AB consists of two consecutive subparts $T A$ and $T B$. $T A$ denotes a set of melody sequences generated by a set of chord-sets $A$ while $T B$ denotes a set of sequences generated by a set of chord-sets $B$. There is no common chord-sets between $T A$ and $T B$. TA-100,000 indicates that the size of the tested window in $T A$ is 100,000 melody sequences. Due to the space limitation, we only discuss the adaptability of the proposed algorithm in this section.

We use the coverage rate [4] to evaluate the adaptability of the MQS-change algorithm. The result is shown in Fig. 6. As the size of a window becomes smaller, the MQS-change adapts more rapidly the change of recent information between the two different subparts of T5.I4.D1000K-AB.

\section{CONCLUSIONS}

In this paper, we propose a new online algorithm MQS-change (changes of Music Query Streams) to maintain two music melody structures (sets of chord-sets and string of chord-sets) and to detect three music melody structure changes (significant pattern bursts, increasing changed patterns and decreasing changed patterns) from a continuous user-centered music query stream. A new summary data structure MSC-list (a list of Music Structure Changes) is developed to maintain the essential information about the maximal melody structures of music query streams so far. Based on our knowledge, MQSchange algorithm is the first online, single-pass method to detect the changes in a continuous user-centered music query stream.

\section{REFERENCES}

[1] R. Agrawal and R. Srikant, "Fast algorithms for mining association rules," in: Proc. VLDB, pp. 487-499, 1994.

[2] B. Babcock, S. Babu, M. Data, R. Motwani and J. Widom, "Models and issues in data stream systems," in: Proc. PODS, pp. $1-16,2002$.

[3] V. Bakhmutora, V. U. Gusev and T.N. Titkova, "The search for adaptations in song melodies," Computer Music Journal, 21 (1), 58-67, 1997.

[4] J. H. Chang and W. S. Lee, "Finding recent frequent itemsets adaptively over online data streams," in: Proc. SIGKDD, pp. 487492, 2003

[5] G. Dong, J. Han, L.V.S. Lakshmanan, J. Pei, H. Wang and P.S. $\mathrm{Yu}$, "Online mining of changes from data streams: research problems and preliminary results," in: Proc. ACM SIGMODMPDS, 2003.

[6] M. M. Gaber, A. Zaslavsky and S. Krishnaswamy, "Mining data streams: a review," ACM SIGMOD Record, 34(1), June 2005. [7] J.-L. Hsu, C.-C. Liu and A.L.P. Chen, "Discovering nontrivial repeating patterns in music data," IEEE Transactions on Multimedia, 3 (3), 311-325, 2001.

[8] G.T. Jones, Music Theory. Harper \& Row, Publishers, New York., 1974.

[9] H.-F. Li, S.-Y. Lee and M.-K. Shan, "Mining frequent closed structures in streaming melody sequences," in: Proc. ICME, 2004.

[10] H.-F. Li, S.-Y. Lee and M.-K. Shan, "Online mining maximal frequent structures in continuous landmark melody streams," Pattern Recognition Letters, 26 (11), 1658-1674, August 2005.

[11] H.-F. Li, S.-Y. Lee and M.-K. Shan, "Online mining changes of items over continuous append-only and dynamic data streams," Journal of Universal Computer Sciences, 11(8), 1411-1425, 2005.

[12] M.-K. Shan and F.-F. Kuo, "Music style mining and classification by melody," IEICE Transactions on Information and Systems, E86-D (4), 655-659, 2003.

[13] A. Yoshitaka and T. Ichikawa, "A survey on content-based retrieval for multimedia databases," IEEE Transactions on Knowledge and Data Engineering, 11 (1), 81-93, 1999.

[14] www.music.com.tw

[15] www.kkbox.com.tw 